\title{
The end of the curve complex
}

\author{
Saul Schleimer
}

\begin{abstract}
Suppose that $S$ is a surface of genus two or more, with exactly one boundary component. Then the curve complex of $S$ has one end.
\end{abstract}

Mathematics Subject Classification (2010). 32G15, 57M99, 30 F99.

Keywords. Curve complex.

\section{Introduction}

We denote the compact, connected, orientable surface of genus $g$ with $b$ boundary components by $S_{g, b}$. The complexity of $S=S_{g, b}$ is $\xi(S):=3 g-3+b$. A simple closed curve $\alpha$ in $S$ is essential if $\alpha$ does not cut a disk out of $S$. Also, $\alpha$ is non-peripheral if it does not cut an annulus out of $S$.

When $\xi(S) \geq 2$ the complex of curves, $\mathcal{C}(S)$, is the simplicial complex where vertices are isotopy classes of essential non-peripheral curves. The $k$-simplices are collections of $k+1$ distinct vertices having disjoint representatives. We regard every simplex as a Euclidean simplex of side-length one. If $\alpha$ and $\beta$ are vertices of $\ell(S)$ let $d_{S}(\alpha, \beta)$ denote the distance between $\alpha$ and $\beta$ in the one-skeleton $\ell^{1}(S)$. It is a pleasant exercise to prove that $\ell(S)$ is connected. It is an important theorem of H. Masur and Y. Minsky [8] that $\ell(S)$ is Gromov hyperbolic.

Let $B(\omega, r):=\left\{\alpha \in \mathcal{C}^{0}(S) \mid d_{S}(\alpha, \omega) \leq r\right\}$ be the ball of radius $r$ about the vertex $\omega$. We will prove:

Theorem 5.1. Fix $S:=S_{g, 1}$ for some $g \geq 2$. For any vertex $\omega \in C(S)$ and for any $r \in \mathbb{N}$ : the subcomplex spanned by $\mathcal{C}^{0}(S) \backslash B(\omega, r)$ is connected.

For such surfaces, Theorem 5.1 directly answers a question of Masur's. It also answers a question of G. Bell and K. Fujiwara [1] in the negative: the complex of curves need not be quasi-isometric to a tree. Theorem 5.1 is also evidence for a positive answer to a question of P. Storm: 
Question 1.1. Is the Gromov boundary of $\mathcal{C}(S)$ connected? ${ }^{1}$

Note that Theorem 5.1 is only evidence for, and not an answer to, Storm's question: for example, there is a one-ended hyperbolic space where the Gromov boundary is a pair of points. Finally, as we shall see in Remark 4.2, it is not obvious how to generalize Theorem 5.1 to surfaces with more (or fewer) boundary components.

Acknowledgments. I thank both Jason Behrstock and Christopher Leininger; their observations, here recorded as Proposition 4.3, were the origin of my thoughts leading to Theorem 5.1. I also thank Kenneth Bromberg for showing me a simplification, given below, of my original proof of Theorem 5.1. Finally, I am grateful to Howard Masur for both posing the main question and for many enlightening conversations.

\section{Definitions and necessary results}

An important point elided above is how to define $\mathcal{C}(S)$ when $\xi(S)=1$. The complex as defined is disconnected in these cases. Instead we allow a $k$-simplex to be a collection of $k+1$ distinct vertices which have representatives with small intersection. For $S_{1,1}$ exactly one intersection point is allowed while $S_{0,4}$ requires two. In both cases $\mathcal{C}(S)$ is the famous Farey tessellation. Note that $\mathcal{C}\left(S_{0,3}\right)$ is empty. We will not need to consider the other low complexity surfaces: the sphere, the disk, the annulus, and the torus.

A subsurface $X \subset S$ is essential if every component of $\partial X$ is essential in $S$. We will generally assume that $\xi(X) \geq 1$. A pair of curves, or a curve and a subsurface, are tight if they cannot be isotoped to reduce intersection. We will generally assume that all curves and subsurfaces discussed are tight with respect to each other. We say a curve $\alpha$ cuts $X$ if $\alpha \cap X \neq \emptyset$. If $\alpha \cap X=\emptyset$ then we say $\alpha$ misses $X$.

Following Masur and Minsky [9], we define the subsurface projection map $\pi_{X}$ : this maps vertices of $\mathcal{C}(S)$ to collections of vertices of $\mathcal{C}(X)$. Fix a vertex $\alpha \in \mathcal{C}(S)$

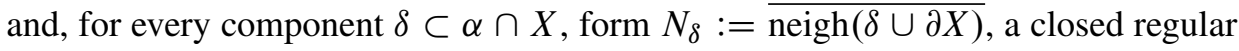
neighborhood of $\delta \cup \partial X$. Take $\pi_{X}(\alpha)$ to be the set of all vertices of $\mathcal{C}(X)$ which appear as a boundary component of some $N_{\delta}$. If $\alpha$ misses $X$ then $\pi_{X}(\alpha)=\emptyset$. Note if $\alpha \subset S$ is contained in $X$ after tightening then $\pi_{X}(\alpha)=\{\alpha\}$.

As a useful bit of notation, if $\alpha$ and $\beta$ both cut $X$, we set

$$
d_{X}(\alpha, \beta):=\operatorname{diam}_{X}\left(\pi_{X}(\alpha) \cup \pi_{X}(\beta)\right)
$$

with diameter computed in $\ell^{1}(X)$. Masur and Minsky give a combinatorial proof (Lemma 2.2 in [9]) that:

\footnotetext{
${ }^{1}$ Leininger and I answered Storm's question in many cases [7]. The complete conjecture has been verified by D. Gabai [3]. The material in this note is of independent interest as it is self-contained and elementary.
} 
Lemma 2.1. If $\alpha$ and $\beta$ are disjoint and both cut $X$ then $d_{X}(\alpha, \beta) \leq 2$.

By geodesic in $\mathcal{C}(S)$ we will always be referring to a geodesic in the one-skeleton. Since $\mathcal{C}(S)$ is Gromov hyperbolic the exact position of the geodesic is irrelevant; we often use the notation $[\alpha, \beta]$ as if the geodesic was determined by its endpoints. We immediately deduce from Lemma 2.1 :

Lemma 2.2. Suppose that $\alpha, \beta$ are vertices of $\mathcal{C}(S)$, both cutting $X$. Suppose that $d_{X}(\alpha, \beta)>2 \cdot d_{S}(\alpha, \beta)$. Then every geodesic $[\alpha, \beta] \subset \mathcal{C}(S)$ has a vertex which misses $X$.

This is essentially Lemma 2.3 of [9].

Remark 2.3. There is a useful special case of Lemma 2.2: assume all the hypotheses and in addition that $\gamma$ is the unique vertex of $\mathcal{C}(S)$ missing $X$. Then every geodesic connecting $\alpha$ to $\beta$ contains $\gamma$.

In fact, $\gamma$ is the unique vertex missing $X$ exactly when $S \backslash \operatorname{neigh}(\gamma)=X$ or $S \backslash \operatorname{neigh}(\gamma)=X \cup P$ with $P \cong S_{0,3}$ : a pair of pants.

Remark 2.4. Note that Lemma 2.2 is a weak form of the Bounded Geodesic Image Theorem ([9], Theorem 3.1). The proof of the stronger result appears to require techniques from Teichmüller theory.

We now turn to the mapping class group $\mathcal{M C} \mathcal{Y}(S)$ : the group of isotopy classes of homeomorphisms of $S$. Note that the natural action of $\mathcal{M} \mathcal{E}(S)$ on $\mathcal{C}(S)$ is via isometries. We have an important fact:

Lemma 2.5. If $\psi: S \rightarrow S$ is a pseudo-Anosov and $\alpha$ is a vertex of $\mathcal{C}^{0}(S)$ then $\operatorname{diam}_{S}\left\{\psi^{n}(\alpha) \mid n \in \mathbb{Z}\right\}$ is infinite.

It follows that the diameter of $\mathcal{C}(S)$ is infinite whenever $\xi(S) \geq 1$. A proof of Lemma 2.5, relying on Kobayashi's paper [5], may be found in the remarks following Lemma 4.6 of [9]. As a matter of fact, Masur and Minsky there prove more using train track machinery: any orbit of a pseudo-Anosov map is a quasi-geodesic. We will not need this sharper version.

Note that if $\psi: S \rightarrow S$ is a homeomorphism then we may restrict $\psi$ to the curve complex of a subsurface $\psi \mid X: \mathcal{C}(X) \rightarrow \mathcal{C}(\psi(X))$. This restriction behaves well with respect to subsurface projection: that is, $\pi_{\psi(X)} \circ \psi=\psi \mid X \circ \pi_{X}$.

We conclude this discussion by examining partial maps. Suppose that $X \subset S$ is an essential surface, not homeomorphic to $S$. If $\psi: S \rightarrow S$ has the property that $\psi|S \backslash X=\operatorname{Id}| S \backslash X$ then we call $\psi$ a partial map supported on $X$. Note that if $\psi$ is supported on $X$ then the orbits of $\psi$ do not have infinite diameter in $\mathcal{C}(S)$. Since $\psi$ fixes $\partial X$ and acts on $\mathcal{C}(S)$ via isometry, every point of an orbit has the same distance to $\partial X$ in $\mathcal{C}(S)$. Nonetheless, Lemmas 2.2 and 2.5 imply: 
Lemma 2.6. Suppose $\psi: S \rightarrow S$ is supported on $X$ and $\psi \mid X$ is pseudo-Anosov. Fix a vertex $\sigma \in \mathcal{C}(S)$ and define $\sigma_{n}:=\psi^{n}(\sigma)$. Then for any $K \in \mathbb{N}$ there is a power $n \in \mathbb{Z}$ so that $d_{X}\left(\sigma, \sigma_{n}\right) \geq K$. In particular, if $K>4 \cdot d_{S}(\sigma, \partial X)$ then every geodesic $\left[\sigma, \sigma_{n}\right] \subset \mathcal{C}(S)$ contains a vertex which misses $X$.

\section{No dead ends}

We require a pair of tools in order to prove Theorem 5.1. The first is:

Proposition 3.1. Fix $S=S_{g, b}$. For any vertex $\omega \in \mathcal{C}(S)$ and for any $r \in \mathbb{N}$ : every component of the subcomplex spanned by $\complement^{0}(S) \backslash B(\omega, r)$ has infinite diameter.

A more pithy phrasing might be: the complex of curves has no dead ends. Proposition 3.1 allows us to push vertices away from $\omega$ while remaining inside the same component of $\mathcal{C}(S) \backslash B(\omega, r)$. The proof is a bit subtle due to the behavior of $\mathcal{C}(S)$ near a non-separating curve.

Proof of Proposition 3.1. If $S=S_{0,3}$ is a pants then the curve complex is empty and there is noting to prove. If $\mathcal{C}(S)$ is a copy of the Farey graph then the claim is an easy exercise. So we may suppose that $\xi(S) \geq 2$.

Now fix a vertex $\alpha \in \mathcal{C}(S) \backslash B(\omega, r)$. Set $n:=d_{S}(\alpha, \omega)$. Thus $n>r$. Our goal is to find a curve $\delta$, connected to $\alpha$ in the complement of $B(\omega, n-1)$, with $d_{S}(\delta, \omega)=n+1$. Doing this repeatedly proves the proposition. Note that finding such a vertex $\delta$ is straight-forward if $r=0$ and $n=1$. This is because $\ell(S) \backslash \omega$ is connected and because, following Lemma 2.5 , we know that the diameter of $\mathcal{C}(S)$ is infinite. Henceforth we will assume that $n \geq 2$; that is, $\omega$ cuts $\alpha$.

Fix attention on a component $X$ of $S \backslash$ neigh $(\alpha)$ which is not a pair of pants. So $\xi(X) \geq 1$ and, by the comments following Lemma $2.5, \mathcal{C}(X)$ has infinite diameter. Since $\omega$ cuts $\alpha$ we find that $\omega$ also cuts $X$. Choose a curve $\beta$ contained in $X$ with $d_{X}(\beta, \omega) \geq 2 n+1$. Note that $d_{S}(\alpha, \beta)=1$. We may assume that $\beta$ is either non-separating or cuts a pants off of $S$. (To see this: if $\beta$ cannot be chosen to be nonseparating then $X$ is planar. As $\xi(X) \geq 1$ we deduce that $X$ has at least four boundary components. At most two of these are parallel to $\alpha$.) It follows from Lemma 2.2 that any geodesic from $\beta$ to $\omega$ in $\ell(S)$ has a vertex $\gamma$ which misses $X$.

By the triangle inequality $d_{S}(\gamma, \omega)$ equals $n$ or $n-1$. In the former case we are done: simply take $\delta=\beta$ and notice that $d_{S}(\beta, \omega)=n+1$. In the latter case $d_{S}(\beta, \omega)=n$ and we proceed as follows: replace $\alpha$ by $\beta$ and replace $X$ by $Z:=S \backslash \operatorname{neigh}(\beta)$. We may now choose $\delta$ to be a vertex of $\mathcal{C}(Z)$ with $d_{Z}(\delta, \omega) \geq$ $2 n+1$. As above, any geodesic $[\delta, \omega] \subset \mathcal{C}(S)$ has a vertex which misses $Z$. Since $\beta$ is the unique vertex not cutting $Z$, our Remark 2.3 implies that $\beta \in[\delta, \omega]$. Thus $d_{S}(\delta, \omega)=n+1$ and we are done. 


\section{The Birman short exact sequence}

We now discuss the second tool needed in the proof of Theorem 5.1. Following I. Kra's notation in [6] let $\dot{S}:=S_{g, 1}$ and $S:=S_{g}$ for a fixed $g \geq 2$. Let $\rho: \dot{S} \rightarrow S$ be the quotient map crushing $\partial \dot{S}$ to a point, say $x \in S$. This leads to the Birman short exact sequence:

$$
\pi_{1}(S, x) \rightarrow \mathcal{M} \mathscr{E}(\dot{S}) \rightarrow \mathcal{M} \mathscr{E}(S)
$$

for $g \geq 2$. The map $\rho$ gives the second arrow. The first arrow is defined by sending $\gamma \in \pi_{1}\left(S, x_{0}\right)$ to a mapping class $\psi_{\gamma}$. There is a representative of this class which is isotopic to the identity, in $S$, via an isotopy dragging $x$ along the path $\gamma$. See Birman's book [2] or Kra's paper [6] for further details.

Fix an essential subsurface $\dot{X} \subset \dot{S}$ and let $X:=\rho(\dot{X})$. If $\gamma \in \pi_{1}(S, x)$ is contained in $X$ then $\psi_{\gamma}$ is a partial map, supported in $\dot{X}$. We say that $\gamma$ fills $X$ if $\gamma \subset X$ and, in addition, every representative of the free homotopy class of $\gamma$ cuts $X$ into a collection of disks and peripheral annuli. For future use we record a well-known theorem of Kra [6]:

Theorem 4.1. Suppose that $\xi(\dot{X}) \geq 1$. If $\gamma$ fills $X$ then $\psi_{\gamma} \mid \dot{X}$ is pseudo-Anosov.

Now note that, corresponding to the Birman short exact sequence, there is a "fibre bundle" of curve complexes:

$$
\mathcal{F}_{\tau} \rightarrow \varphi(\dot{S}) \rightarrow \digamma(S) .
$$

Here $\tau$ is an arbitrary vertex of $\mathcal{C}(S)$ and $\mathcal{F}_{\tau}:=\rho^{-1}(\tau)$. The second arrow is given by $\rho$. The first is the inclusion of $\mathcal{F}_{\tau}$ into $\mathcal{C}(\dot{S})$.

Remark 4.2. If $|\partial S| \geq 2$ then collapsing one boundary component does not induce a map on the associated curve complexes. Thus, it is not clear how to generalize Theorem 5.1 to such surfaces. If $\partial S$ is empty then it appears to be very difficult to find interesting mapping class group equivariant electrifications of $\mathcal{C}(S)$.

Using the Birman short exact sequence we obtain an action of $\pi_{1}(S, x)$ on the curve complex $\mathcal{C}(\dot{S})$. Behrstock and Leininger observe that:

Proposition 4.3. The map $\rho: \mathcal{C}(\dot{S}) \rightarrow \mathcal{C}(S)$ has the following properties:

- It is 1-Lipschitz.

- For any $\alpha \in \mathcal{C}(\dot{S}), \gamma \in \pi_{1}(S, x)$ we have $\rho(\alpha)=\rho\left(\psi_{\gamma}(\alpha)\right)$.

- Every fibre $\mathcal{F}_{\tau}$ is connected.

Remark 4.4. Behrstock and Leininger's interest in the fibre $\mathcal{F}_{\tau}$ was to give a "natural" subcomplex of $\mathcal{C}(S)$ which is not quasi-convex: this is implied by the first pair of properties. 
Remark 4.5. More of the structure of $\mathscr{F}_{\tau}$ is known. For example, since $S$ is closed, the fibre $\mathscr{F}_{\tau}$ is either a single $\pi_{1}(S, x)$-orbit or the union of a pair of orbits depending on whether $\tau$ is non-separating or separating. Furthermore, $\mathscr{F}_{\tau}$ is a tree. See [4] for a detailed discussion.

Proof of Proposition 4.3. Fix an essential non-peripheral curve $\alpha$ in $\dot{S}$. Note that $\rho(\alpha)$ is essential in $S$ and so the induced map $\rho: \mathcal{C}(\dot{S}) \rightarrow \mathcal{C}(S)$ is well-defined. If $\alpha$ and $\beta$ are disjoint in $\dot{S}$ then so are their images in $S$. Thus $\rho$ does not increase distance between vertices and the first conclusion holds.

Now fix a curve $\alpha \subset \dot{S}$ and $\gamma \in \pi_{1}(S, x)$. Note that $\psi_{\gamma}$ is isotopic to the identity in $S$. Thus the images $\rho\left(\psi_{\gamma}(\alpha)\right)$ and $\rho(\alpha)$ are isotopic in $S$. It follows that $\rho(\alpha)=\rho\left(\psi_{\gamma}(\alpha)\right)$ as vertices of $\mathcal{C}(S)$, as desired.

Finally, fix $\tau \in \mathscr{C}(S)$. Let $\mathscr{F}_{\tau}$ be the fibre over $\tau$. Pick $\alpha, \beta \in \mathscr{F}_{\tau}$. It follows that $a:=\rho(\alpha)$ and $b:=\rho(\beta)$ are both isotopic to $\tau$ and so to each other. We induct on the intersection number $\iota(\alpha, \beta)$. Suppose the intersection number is zero. Then $\alpha$ and $\beta$ are disjoint and we are done. Suppose that the intersection number is non-zero. Since $a$ and $b$ are isotopic, yet intersect, they are not tight with respect to each other. It follows that there is a bigon $B \subset S \backslash(a \cup b)$. Since $\alpha$ and $\beta$ are tight in $\dot{S}$ the point $x$ must lie in $B$. Let $\dot{B}:=\rho^{-1}(\bar{B})$. Now construct a curve $\beta^{\prime} \subset \dot{S}$ by starting with $\beta$, deleting the $\operatorname{arc} \beta \cap \dot{B}$, and adding the $\operatorname{arc} \alpha \cap \dot{B}$. Isotope $\beta^{\prime}$ to be tight with respect to $\alpha$. Now $\beta^{\prime} \in \mathscr{F}_{\tau}$ because $\rho\left(\beta^{\prime}\right)$ is isotopic to $\rho(\beta)$ in $S$. Finally, $\iota\left(\alpha, \beta^{\prime}\right) \leq \iota(\alpha, \beta)-2$.

\section{Proving the theorem}

We are now equipped to prove:

Theorem 5.1. Fix $\dot{S}:=S_{g, 1}$ for some $g \geq 2$. For any vertex $\omega \in C(\dot{S})$ and for any $r \in \mathbb{N}$ : the subcomplex spanned by $\complement^{0}(\dot{S}) \backslash B(\omega, r)$ is connected.

As above we use the notation $\dot{S}=S_{g, 1}$ and $S=S_{g}$ for some fixed $g \geq 2$. Also, we have defined a map $\rho: \mathcal{\ell}(\dot{S}) \rightarrow \ell(S)$ induced by collapsing $\partial \dot{S}$ to a point, $x$. As above we use $\mathscr{F}_{\tau}=\rho^{-1}(\tau)$ to denote the fibre over $\tau$.

Proof of Theorem 5.1. Choose $\alpha^{\prime}$ and $\beta^{\prime}$ vertices of $\mathcal{C}(\dot{S}) \backslash B(\omega, r)$. By Proposition 3.1 we may connect $\alpha^{\prime}$ and $\beta^{\prime}$, by paths disjoint from $B(\omega, r)$, to vertices outside of $B(\omega, 3 r)$. Call these new vertices $\alpha$ and $\beta$. We may assume that both $\alpha$ and $\beta$ are non-separating because such vertices are 1-dense in $\mathcal{C}(\dot{S})$.

Choose any vertex $\tau \in \mathscr{C}(S)$ so that $d_{S}(\tau, \rho(\omega)) \geq 4 r$. This is always possible because $\mathcal{C}(S)$ has infinite diameter. (See the remarks after Lemma 2.5.) It follows from Proposition 4.3 that $\mathcal{F}_{\tau} \cap B(\omega, r)=\emptyset$. We will now connect each of $\alpha$ and $\beta$ to some point of $\mathscr{F}_{\tau}$ via a geodesic disjoint from $B(\omega, r)$. Since $\mathscr{F}_{\tau}$ is connected, by Proposition 4.3, this will complete the proof of Theorem 5.1. 
Let $\dot{X}:=\dot{S} \backslash \alpha$ and take $X:=\rho(\dot{X})$. Fix any point $\sigma$ in $\mathscr{F}_{\tau}$. If $\sigma=\alpha$ then $\alpha$ is trivially connected to the fibre. So suppose that $\sigma \neq \alpha$. Since $\alpha$ is non-separating deduce that $\sigma$ cuts $\dot{X}$. Now, since $\xi(\dot{S}) \geq 4$ we have $\xi(\dot{X}) \geq 3$. Let $\gamma \in \pi_{1}(S, x)$ be any homotopy class so that $\psi_{\gamma}$ is supported in $\dot{X}$ and so that $\gamma$ fills $X$. By Kra's Theorem (4.1) $\psi_{\gamma} \mid \dot{X}$ is pseudo-Anosov.

Since $\mathcal{F}_{\tau}$ is left setwise invariant by $\pi_{1}(S, x)$ (Proposition 4.3) the curves $\sigma_{n}:=$ $\psi_{\gamma}^{n}(\sigma)$ all lie in $\mathscr{F}_{\tau}$. Since $\psi_{\gamma} \mid \dot{X}$ is pseudo-Anosov, Lemma 2.6 gives an $n \in \mathbb{Z}$ so that every geodesic $g:=\left[\sigma, \sigma_{n}\right] \subset \mathcal{C}(\dot{S})$ has a vertex which misses $\dot{X}$. Since $\alpha$ is non-separating, as in Remark 2.3, it follows that $\alpha$ is actually a vertex of $g$.

We now claim that at least one of the two segments $[\sigma, \alpha] \subset g$ or $\left[\alpha, \sigma_{n}\right] \subset g$ avoids the ball $B(\omega, r)$. For suppose not: then there are vertices $\mu, \mu^{\prime} \in g$ on opposite sides of $\alpha$ which both lie in $B(\omega, r)$. Thus $d_{\dot{S}}\left(\mu, \mu^{\prime}\right) \leq 2 r$. Since $g$ is a geodesic the length along $g$ between $\mu$ and $\mu^{\prime}$ is at most $2 r$. Thus $d_{\dot{S}}(\omega, \alpha) \leq 2 r$. This is a contradiction.

Thus we can connect $\alpha$ to a vertex of $\mathcal{F}_{\tau}$ (namely, $\sigma$ or $\sigma_{n}$ ) avoiding $B(\omega, r)$. Identically, we can connect $\beta$ to a vertex of $\mathscr{F}_{\tau}$ while avoiding $B(\omega, r)$. As noted above, this completes the proof.

\section{References}

[1] G. C. Bell and K. Fujiwara, The asymptotic dimension of a curve graph is finite. J. London Math. Soc. (2) 77 (2008), 33-50. Zbl 1135.57010 MR 2389915

[2] J. S. Birman, Braids, links, and mapping class groups. Ann. of Math. Stud. 82, Princeton University Press, Princeton, N.J., 1974. Zbl 0305.57013 MR 0375281

[3] D. Gabai, Almost filling laminations and the connectivity of ending lamination space. Geom. Topol. 13 (2009), 1017-1041. Zbl 1165.57015 MR 2470969

[4] R. P. Kent, IV, C. J. Leininger, and S. Schleimer, Trees and mapping class groups. J. Reine Angew. Math. 637 (2009), 1-21. Zbl 1190.57014 MR 2599078

[5] T. Kobayashi, Heights of simple loops and pseudo-Anosov homeomorphisms. In Braids (Santa Cruz, CA, 1986), Contemp. Math. 78, Amer. Math. Soc., Providence, RI, 1988, 327-338. Zbl 0663.57010 MR 0975087

[6] I. Kra, On the Nielsen-Thurston-Bers type of some self-maps of Riemann surfaces. Acta Math. 146 (1981), 231-270. Zbl 0477.32024 MR 611385

[7] C. J. Leininger and S. Schleimer, Connectivity of the space of ending laminations. Duke Math. J. 150 (2009), 533-575. Zbl 1190.57013 MR 2582104

[8] H. A. Masur and Y. N. Minsky, Geometry of the complex of curves I: Hyperbolicity. Invent. Math. 138 (1999), 103-149. Zbl 0941.32012 MR 1714338

[9] H. A. Masur and Y. N. Minsky, Geometry of the complex of curves II: Hierarchical structure. Geom. Funct. Anal. 10 (2000), 902-974. Zbl 0972.32011 MR 1791145 
Received August 4, 2007; revised October 18, 2009

S. Schleimer, Department of Mathematics, Rutgers University, 110 Frelinghuysen Rd., Piscataway, NJ 08854-8019, U.S.A.

E-mail: saulsch@math.rutgers.edu 